\title{
A Case Report on Premature Twins: Primary Congenital Glaucoma or Large Cupping Disks Mimicking Primary Congenital Glaucoma?
}

\author{
Shunsuke Nakakura ${ }^{1}$, Etsuko Terao ${ }^{1}$, Nanami Kuroda ${ }^{1}$, Shota Fujio ${ }^{1}$, Yuka Hirose ${ }^{1}$, Akio Tabuchi ${ }^{1}$, \\ Yoshiaki Kiuchi ${ }^{2}$ \\ 1. Ophthalmology, Saneikai Tsukazaki Hospital, Himeji, JPN 2. Ophthalmology, Hiroshima University, Hiroshima, JPN
}

Corresponding author: Shunsuke Nakakura, s.nakakura@tsukazaki-eye.net

\begin{abstract}
Large cupping of the optic disk in a baby or a child can be indicative of primary congenital glaucoma. Primary congenital glaucoma is often refractory to treatment, and lifelong management and follow-up are necessary; therefore, diagnosis requires careful consideration. In this study, we describe the case of twins in whom primary congenital glaucoma was initially suspected due to large cupping of the optic disks. Twin babies (a boy and a girl weighing $455 \mathrm{~g}$ and $592 \mathrm{~g}$ respectively), born prematurely (at 28 gestational weeks), were referred to our hospital eight months after birth because large cupping of the optic disks was detected during follow-up for retinopathy of prematurity. According to color fundus photographs, the cup/disk ratios in both eyes of both babies ranged from 0.75 to 0.86 . However, the axial length ranged from 18.57 to $19.91 \mathrm{~mm}$, the anterior chamber depth ranged from 2.68 to $2.93 \mathrm{~mm}$, and the horizontal diameters of the corneas, which were clear, ranged from 10 to $10.5 \mathrm{~mm}$. The intraocular pressures (IOPs), as measured by a rebound tonometer, were 15.3-19.7 mmHg. Glaucoma was strongly suspected due to the large cupping of the optics disks; however, other ocular biometric tests demonstrated that the eyes were normal. After eight months of follow-up without any medication or intervention, the IOPs stabilized between 6-22.1 $\mathrm{mmHg}$, the refractive errors were between -2.5 and 0 diopters, and we found no apparent enlargement of the optic disks. In addition, we investigated both parents' optic disks to evaluate the genetic factors and found that they had relatively large C/D ratios (0.68-0.79). These premature twins exhibited glaucoma-like optic disks with large cupping, but no solid glaucomatous changes were observed with ocular biometry and IOP testing. We concluded, therefore, that the early birth and lower birth weights may have been associated with the large cupping of the patients' optic disks. To differentiate between normal physiological cupping and primary congenital glaucoma, ocular morphological examination of the eye, IOP measurements, and investigation of the parents' optic disks were useful.
\end{abstract}

Review began 07/28/2021 Review ended 08/01/2021 Published 08/11/2021

\section{() Copyright 2021}

Nakakura et al. This is an open access article distributed under the terms of the Creative Commons Attribution License CC-BY 4.0., which permits unrestricted use, distribution, and reproduction in any medium, provided the original author and source are credited.
Categories: Ophthalmology, Pediatrics

Keywords: primary congenital glaucoma, child glaucoma, preterm, twins, genetics, intraocular pressure

\section{Introduction}

Primary congenital glaucoma (PCG) is a rare variety of glaucoma and commonly presents between the ages of three to nine months; however, the most severe form is newborn-onset [1]. Elevated intraocular pressure (IOP) is associated with the classic "triad" of symptoms (photophobia, epiphora, and blepharospasm) which occurs due to rapid expansion of the eye, causing buphthalmos, corneal enlargement, Haab striae, and subsequent corneal edema and opacification [1]. If the IOP increases consistently, optic cup enlargement will progress.

In this study, we describe the case of twins in whom PCG was initially suspected due to large cupping of the optic disks in both eyes of both children. In this case report, we describe how to distinguish between PCG and normal physiological cupping.

\section{Case Presentation}

This non-interventional, retrospective study received approval from the Institutional Review Board of Saneikai Tsukazaki Hospital (No. 211019) and was performed according to the Declaration of Helsinki.

Premature (born at 28 gestational weeks) twins (a 455-g boy and a 592-g girl) were referred to our hospital eight months after birth because large cupping of the optic disks was found during follow-up for retinopathy of prematurity. During their first visit to our hospital, we investigated the babies carefully without sedatives. They had no signs of the classic "triad" of symptoms of PCG (photophobia, epiphora, and blepharospasm). In the boy, hand-slit lamp examination showed no apparent Haab's striae, no corneal edema, no conjunctival injection, and normal anterior chamber depth (i.e., slightly shallower than that of adults). His horizontal corneal diameters were $10 \mathrm{~mm}$ in the right eye and $10.5 \mathrm{~mm}$ in the left eye. IOP, measured by an IcarePRO rebound tonometer (Icare Finland Oy, Helsinki, Finland), was $19.7 \mathrm{mmHg}$ in the right eye and 16.9 $\mathrm{mmHg}$ in the left. Axial length and anterior chamber depth, measured by ultrasound (UD-8000AB, Tomey, 


\section{Cureus}

Nagoya, Japan), were 19.91/2.73 $\mathrm{mm}$ in the right eye and 19.52/2.90 $\mathrm{mm}$ in the left eye. In the girl, hand-slit lamp examination also revealed no apparent signs of PCG. Her horizontal corneal diameters were $10.5 \mathrm{~mm}$ in the right eye and $10 \mathrm{~mm}$ in the left eye. IOP was $18.9 \mathrm{mmHg}$ in the right eye and $15.3 \mathrm{mmHg}$ in the left. The axial length and anterior chamber depth were $18.57 / 2.93 \mathrm{~mm}$ in the right eye and $18.81 / 2.68 \mathrm{~mm}$ in the left.

After obtaining the ocular biometry measurements, we took color fundus photographs using a wide-field digital camera (RetCam 3, Natus, Pleasanton, California, USA) while the patients' pupils were dilated. Figure 1 shows the color fundus photographs with magnified optic disk and facial photographs. In the boy patient (Figure $1 \mathrm{~A}$ ), the vertical and horizontal cup/disk ratio (C/D ratio) was $0.77 / 0.76$ in the right eye and 0.81/0.76 in the left eye. In the girl patient (Figure 1B), the vertical and horizontal C/D ratio was $0.86 / 0.78$ in the right eye and $0.75 / 0.83$ in the left eye.
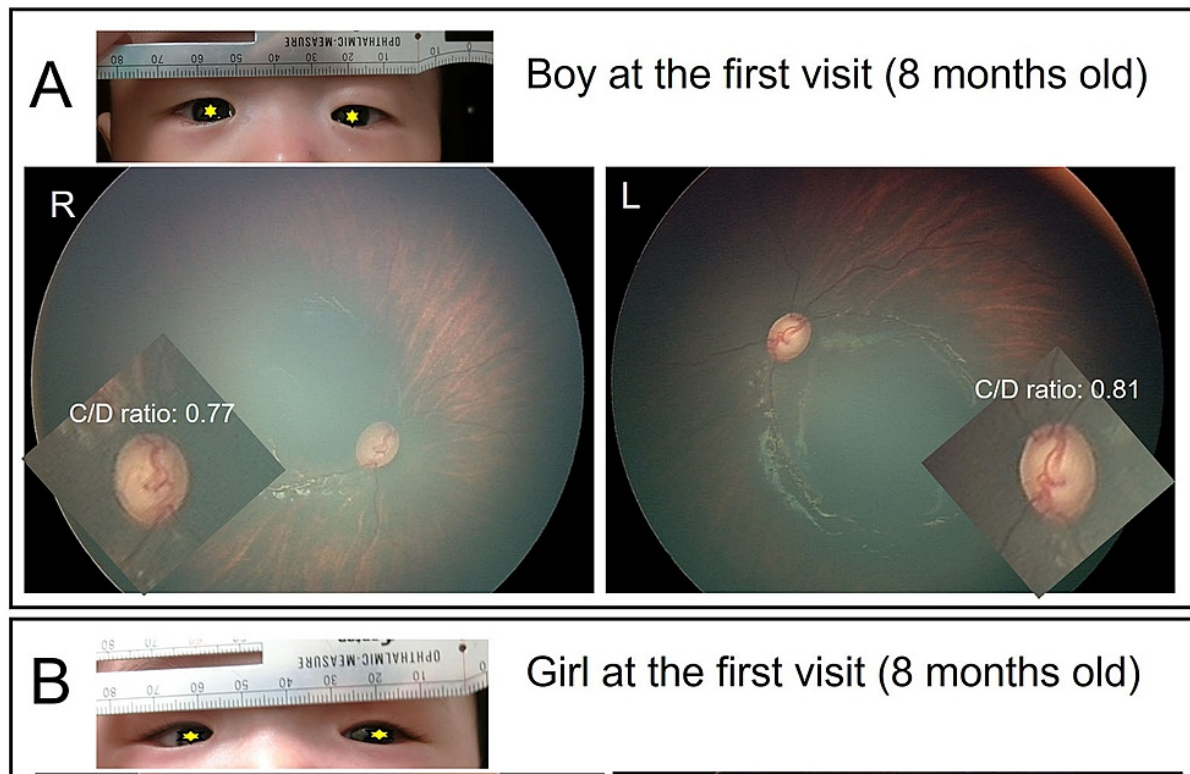

Girl at the first visit (8 months old)
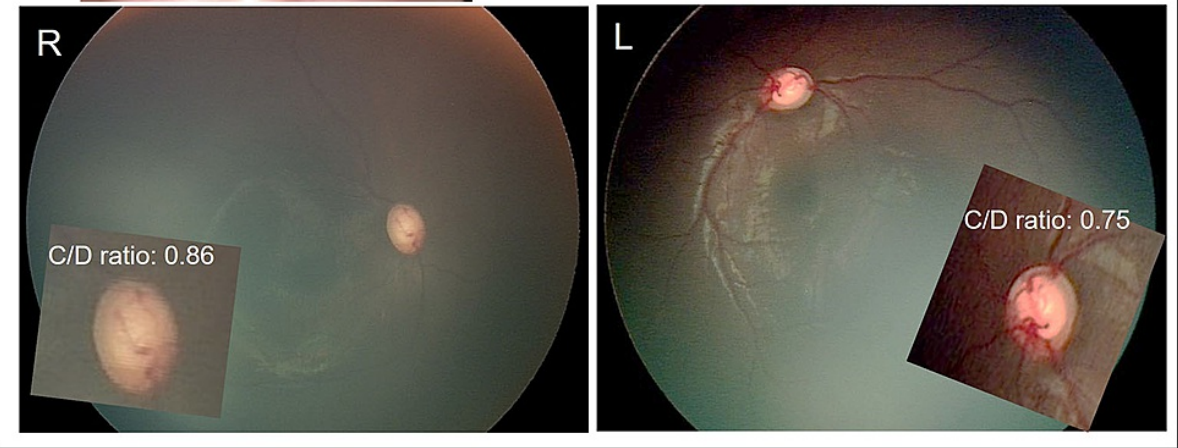

FIGURE 1: Color fundus photography and facial photographs at the first visit (eight months of age)

For their first examination during their first visit, the ocular biometry and IOP results were normal for babies of their age. However, the fundus color photographs showed large cupping of the optic disks (vertical C/D ratios ranged from 0.75 to 0.86 ), strongly suggesting glaucoma. If PCG is suspected, it may be desirable to perform an examination under general anesthesia. However, we could, fortunately, measure IOP without crying using a rebound tonometer, and obtain almost the necessary information except for central corneal thickness and gonioscopic photography. We consulted specialists in child glaucoma (authors Y.K. and A.T.) and followed up carefully without any drugs or intervention.

The results of all the examinations at each visit are shown in Table 1. At 16 months of age, the babies' horizontal corneal diameters did not change remarkably. The axial lengths and anterior chamber depths increased slightly. However, the spherical equivalent measured by Spot ${ }^{\mathrm{TM}}$ Vision Screener (Welch Allyn, New York, America) showed no apparent myopic shift. Additionally, the IOPs were stable at under $22 \mathrm{mmHg}$ during the follow-up period. Fundus photography captured by Retcam at 16 months (Figure 2) showed no obvious enlargement of the $\mathrm{C} / \mathrm{D}$ ratio in either child. 


\section{Cureus}

\begin{tabular}{|c|c|c|c|c|}
\hline \multirow[b]{2}{*}{ Eye } & \multicolumn{2}{|l|}{ Boy } & \multicolumn{2}{|l|}{ Girl } \\
\hline & Right eye & Left eye & Right eye & Left eye \\
\hline \multicolumn{5}{|l|}{$\mathrm{C} / \mathrm{D}$ ratio } \\
\hline Vertical C/D ratio at 8 months of age & 0.77 & 0.81 & 0.86 & 0.75 \\
\hline Vertical C/D ratio at 16 months of age & 0.76 & 0.81 & 0.7 & 0.72 \\
\hline Horizontal C/D ratio at 8 months of age & 0.76 & 0.76 & 0.78 & 0.83 \\
\hline Horizontal C/D at 16 months of age & 0.77 & 0.76 & 0.85 & 0.82 \\
\hline \multicolumn{5}{|l|}{ Axial length and anterior chamber depth (mm) } \\
\hline Axial length at 8 months of age & 19.91 & 19.52 & 18.57 & 18.81 \\
\hline Axial length at 16 months of age & 20.82 & 20.9 & 19.97 & 19.73 \\
\hline ACD at 8 months of age & 2.73 & 2.90 & 2.93 & 2.68 \\
\hline ACD at 16 months of age & 2.98 & 3.16 & 3.32 & 3.22 \\
\hline \multicolumn{5}{|l|}{ Corneal diameter $(\mathrm{mm})$} \\
\hline Horizontal corneal diameter at 8 months of age & 10 & 10.5 & 10.5 & 10 \\
\hline Horizontal corneal diameter at 1 year of age & 11 & 10 & 10 & 10 \\
\hline Horizontal corneal diameter at 16 months of age & 11 & 11 & 10 & 11 \\
\hline \multicolumn{5}{|l|}{ Refraction (diopter) } \\
\hline Spherical equivalent at 8 months of age & -2.5 & -0.25 & 0 & 0.25 \\
\hline Spherical equivalent at 9.5 months of age & -1.25 & -0.75 & -0.25 & -0.5 \\
\hline Spherical equivalent at 1 year of age & -1.25 & -0.25 & -1.75 & -1 \\
\hline Spherical equivalent at 16 months of age & 0.75 & 1.25 & 0 & 0.75 \\
\hline \multicolumn{5}{|l|}{ IOP (mmHg) } \\
\hline IOP at 8 months of age & 19.7 & 16.9 & 18.9 & 15.3 \\
\hline IOP at 8.5 months of age & 22.1 & 21.2 & 21.6 & 22.1 \\
\hline IOP at 9.5 months of age & 21.6 & 21.7 & 16 & 22.6 \\
\hline IOP at 1 year of age & 18 & 18 & 10 & 11 \\
\hline IOP at 16 months of age & 14 & 16 & 9 & 6 \\
\hline
\end{tabular}

TABLE 1: Patients' data during follow-up 


\section{Cureus}
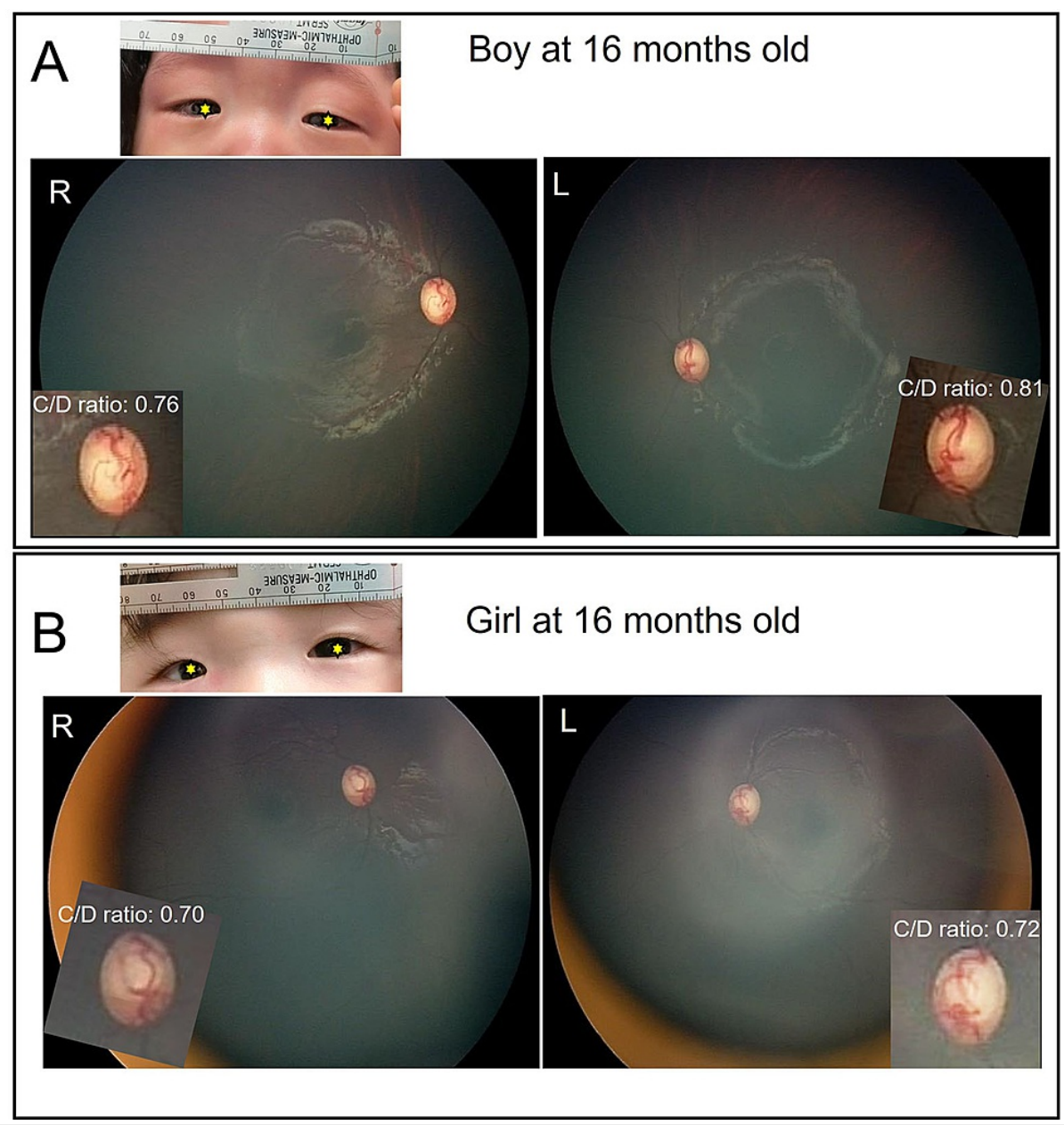

FIGURE 2: Color fundus photography and facial photographs at 16 months of age

We evaluated the parents' optic disks using optical coherence tomography (DRI OCT Triton, Topcon Corporation, Nagoya, Japan) to find out whether genetics factors were involved. As a result, OCT showed that both parents had large C/D ratios (0.68-0.79) (Figure 3).

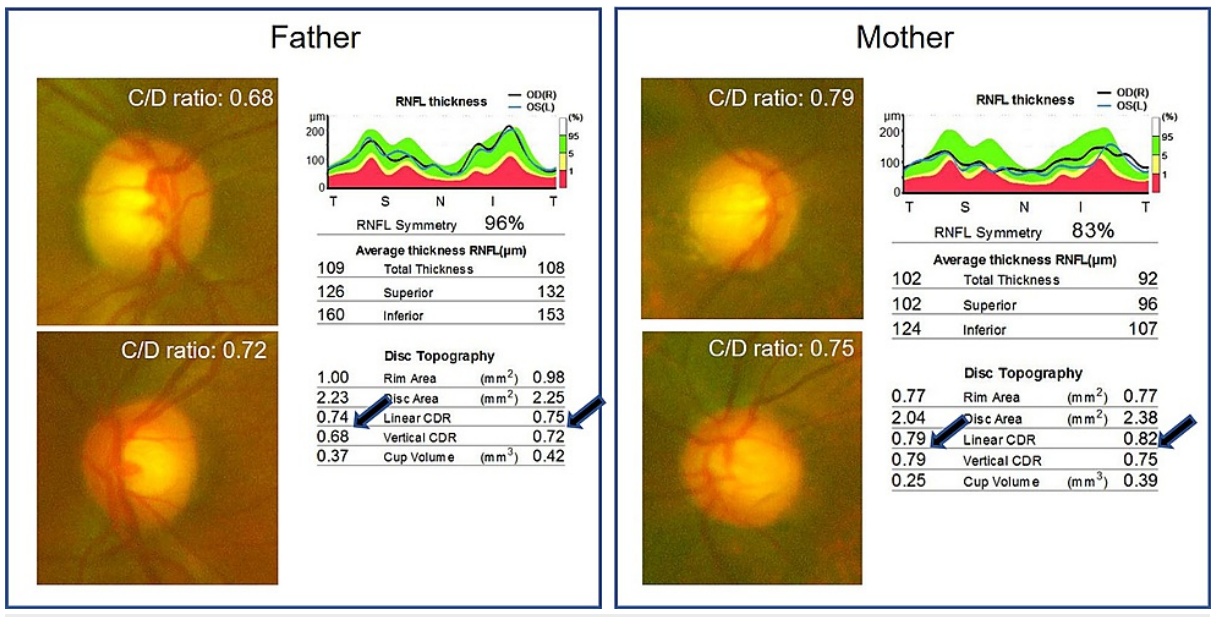

FIGURE 3: Optical coherence tomography (OCT) analysis in the parents

\section{Discussion}

Our cases showed no apparent ocular biometry changes and a normal IOP range during the follow-up period. 
Fortunately, signs such as corneal edema, enlargement of the eye with buphthalmos, and Haab striae due to high IOP were not found. According to a previous study into child glaucoma [2], a corneal diameter greater than $13 \mathrm{~mm}$ in a child of any age is abnormal, and corneal enlargement due to IOP occurs before three years of age. Additionally, the normal axial length at 15 months is between 20 and $22.6 \mathrm{~mm}$ (95\% confidence interval) [2]; therefore, the data of our cases were within the normal ranges. Meanwhile, a C/D ratio of $>0.3$ in a Caucasian infant younger than one year and $>0.5$ in an older child can indicate glaucoma [2]. Only optic disk appearance looks unlike normal baby in our cases. We searched previous studies in PubMed using the keywords "preterm infant" and "cup disk ratio" or "large cupping disk" or "large cup disk" and found 14 papers published between 1978 and 2018 [3-16]. Six papers reported that the C/D ratio is larger in premature infants than in full-term infants $[4,5,7,9,10,15]$. Three papers suggested that the C/D ratio is similar in preterm babies and full-term babies $[3,6,8]$. Therefore, the idea that premature infants have larger cupping disks was predominant. Wikstrand's report also supported that a low birth weight (SD score) and a low weight at week 32 (SD score) were associated with a larger area of the optic cup $(r=-0.37, p=0.0083$, and $r=$ $-0.38, \mathrm{p}=0.0064$, respectively) as well as with a smaller neuronal rim area of the optic nerve head $(\mathrm{r}=0.35$, $\mathrm{p}=0.010$, and $\mathrm{r}=0.31, \mathrm{p}=0.023)[17]$.

However, genetic factors from the parents should be also considered. In our case, neither parent had a history of hereditary optic neuropathy. He et al. reported that the C/D ratio was $78.6 \%$ for genetic and $21.4 \%$ for unshared environmental effects [18]. Park et al. reported that a family history of large C/D ratio was a significant factor associated with a large vertical C/D ratio in children [19]. Han et al. reported that the narrow-sense heritability of the C/D ratio was 47\%-48\% [20]. Healey et al. reported that genetic factors explained $73 \%, 66 \%$, and $34 \%$ of variations in optic disk, cup, and rim areas, respectively [21]. Environmental factors also seem to be important [18,21]. Overall, genetic factors are considered to be important for child optic disk shapes.

\section{Conclusions}

We speculated that with lack of positive family history and the classical "triad" of PCG, the early birth with the low birth weights and the large cupping of the patients' optic disks may have resulted in the optic disks' shapes in the two babies. Further careful long-term follow-up is needed to differentiate between normal physiological large cupping and PCG. Additionally, gonioscopy and visual field tests need to be conducted as the patients grow. Moreover, ocular morphological examination of the eye, IOP measurements, and investigation of the parents' optical disks using fundus photography were useful in helping us differentiate between normal physiological large cupping and PCG in this case.

\section{Additional Information \\ Disclosures}

Human subjects: Consent was obtained or waived by all participants in this study. IRB of Saneikai Tsukazaki Hospital issued approval No. 211019. This non-interventional, retrospective study received approval from the Institutional Review Board of Saneikai Tsukazaki Hospital (No. 211019) and was performed according to the Declaration of Helsinki. Conflicts of interest: In compliance with the ICMJE uniform disclosure form, all authors declare the following: Payment/services info: All authors have declared that no financial support was received from any organization for the submitted work. Financial relationships: All authors have declared that they have no financial relationships at present or within the previous three years with any organizations that might have an interest in the submitted work. Other relationships: All authors have declared that there are no other relationships or activities that could appear to have influenced the submitted work.

\section{References}

1. https://eyewiki.org/Primary_Congenital_Glaucoma.

2. Weinreb RN, Grajewski AL, Papadopoulos M, Grigg J, Freedman S: Child Glaucoma. WGA Consensus Series 9. Kugler Publications, Amsterdam; 2013.

3. Choo MM, Yeong CM, Grigg JR, et al.: Central corneal thickness changes and horizontal corneal diameter in premature infants: A prospective analysis. Medicine (Baltimore). 2018, 97:e13357. 10.1097/MD.0000000000013357

4. Ma Y, Deng G, Ma J, Liu J, Li S, Lu H: Universal ocular screening of 481 infants using wide-field digital imaging system. BMC Ophthalmol. 2018, 18:283. 10.1186/s12886-018-0943-7

5. Kim J, Choi DY, Park KA, Oh SY: Cup-to-disc and arteriole-to-venule ratios in preterm birth. Eye (Lond). 2015, 29:1167-72. 10.1038/eye.2015.99

6. Tong AY, El-Dairi M, Maldonado RS, et al.: Evaluation of optic nerve development in preterm and term infants using handheld spectral-domain optical coherence tomography. Ophthalmology. 2014, 121:1818-26. 10.1016/j.ophtha.2014.03.020

7. Alshaarawi S, Shatriah I, Zunaina E, Wan Hitam WH: Assessment of the optic nerve head parameters using Heidelberg retinal tomography III in preterm children. PLoS One. 2014, 9:e88056. 10.1371/journal.pone.0088056

8. Glass LR, Cioffi GA, Blumberg DM: Retinal nerve fiber layer analysis of cupping in children born prematurely. J Glaucoma. 2014, 23:e1-5. 10.1097/IJG.0000000000000006

9. Park JW, Park SW, Heo H: RetCam image analysis of the optic disc in premature infants . Eye (Lond). 2013, 
27:1137-41. 10.1038/eye.2013.151

10. Hackl S, Zeman F, Helbig H, Oberacher-Velten IM: Optic disc morphology in premature infants. Br J Ophthalmol. 2013, 97:314-7. 10.1136/bjophthalmol-2012-302066

11. Park HJ, Hampp C, Demer JL: Longitudinal study of optic cup progression in children . J Pediatr Ophthalmol Strabismus. 2011, 48:151-6. 10.3928/01913913-20100719-06

12. Pogrebniak AE, Wehrung B, Pogrebniak KL, Shetty RK, Crawford P: Violation of the ISNT rule in nonglaucomatous pediatric optic disc cupping. Invest Ophthalmol Vis Sci. 2010, 51:890-5. 10.1167/iovs.093837

13. McLoone E, O'Keefe M, Donoghue V, McLoone S, Horgan N, Lanigan B: RetCam image analysis of optic disc morphology in premature infants and its relation to ischaemic brain injury. Br J Ophthalmol. 2006, 90:46571. 10.1136/bjo.2005.078519

14. Jacobson L, Hård AL, Svensson E, Flodmark O, Hellström A: Optic disc morphology may reveal timing of insult in children with periventricular leucomalacia and/or periventricular haemorrhage. Br J Ophthalmol. 2003, 87:1345-9. 10.1136/bjo.87.11.1345

15. Yang CM, Liu KR, Chen MS, Ko LS: Ocular fundus examination in premature infants. J Formos Med Assoc. 1991, 90:480-6.

16. Fledelius H: Optic disc cupping and prematurity. Large cups as a possible low birth weight sequel . Acta Ophthalmol (Copenh). 1978, 56:563-73. 10.1111/j.1755-3768.1978.tb01369.x

17. Wikstrand MH, Hård AL, Niklasson A, Hellström A: Birth weight deviation and early postnatal growth are related to optic nerve morphology at school age in children born preterm. Pediatr Res. 2010, 67:325-9. 10.1203/PDR.0b013e3181ca9f43

18. He M, Liu B, Huang W, et al.: Heritability of optic disc and cup measured by the Heidelberg Retinal Tomography in Chinese: the Guangzhou twin eye study. Invest Ophthalmol Vis Sci. 2008, 49:1350-5. 10.1167/iovs.07-1146

19. Park HL, Ha MI, Shin SY: The effect of parental factors in children with large cup-to-disc ratios . PLoS One. 2017, 12:e175900. 10.1371/journal.pone.0175900

20. Han JC, Ko H, Kim SH, et al.: Heritability of the morphology of optic nerve head and surrounding structures: The Healthy Twin Study. PLoS One. 2017, 12:e0187498. 10.1371/journal.pone.0187498

21. Healey P, Carbonaro F, Taylor B, Spector TD, Mitchell P, Hammond CJ: The heritability of optic disc parameters: a classic twin study. Invest Ophthalmol Vis Sci. 2008, 49:77-80. 10.1167/iovs.07-0962 